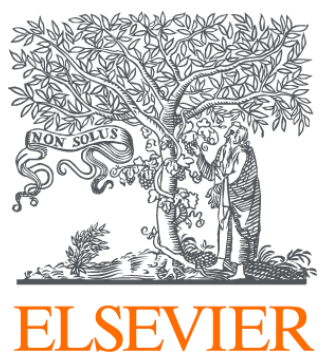

Since January 2020 Elsevier has created a COVID-19 resource centre with free information in English and Mandarin on the novel coronavirus COVID-

19. The COVID-19 resource centre is hosted on Elsevier Connect, the company's public news and information website.

Elsevier hereby grants permission to make all its COVID-19-related research that is available on the COVID-19 resource centre - including this research content - immediately available in PubMed Central and other publicly funded repositories, such as the WHO COVID database with rights for unrestricted research re-use and analyses in any form or by any means with acknowledgement of the original source. These permissions are granted for free by Elsevier for as long as the COVID-19 resource centre remains active. 
Major Article

\title{
The powder keg: Lessons learned about clinical staff preparedness during the early phase of the COVID-19 pandemic
}

\author{
Sainfer Aliyu PhD, MSEd, MHPM, RN ${ }^{\mathrm{a}, *}$, Allison A. Norful PhD, RN, ANP-BC ${ }^{\mathrm{b}}$, Krista Schroeder PhD, RN ${ }^{\mathrm{c}}$, \\ Michelle Odlum EdD, MPH ${ }^{\mathrm{b}}$, Bonnie Glica MSN, RN ${ }^{\mathrm{a}}$, Jasmine L. Travers PhD, RN, AGPCNP-BC ${ }^{\mathrm{d}}$ \\ ${ }^{a}$ MedStar Washington Hospital Center, Washington, DC \\ ${ }^{\mathrm{b}}$ Columbia University School of Nursing, New York, NY \\ ${ }^{\mathrm{c}}$ Temple University College of Public Health, Philadelphia, PA \\ ${ }^{\mathrm{d}}$ New York University Rory Meyers College of Nursing, New York, NY
}

Key Words:

SARS-CoV-2, COVID-19

Health care workers

Health care professionals

Hospital emergency

Hospital preparedness
Background: Little is known about clinical staff's perspectives on preparedness for a pandemic. The purpose
of this study was to obtain various clinical staff perspectives about preparedness to meet the demands for care during the early phase of the SARS-CoV-2 (COVID-19) pandemic.

Methods: We conducted a qualitative study using semistructured in-person interviews from March 2020 to April 2020 at a large tertiary academic urban hospital center. Interview guides were informed by the Resilience Framework for Public Health Emergency Preparedness and analyzed using a directed content analysis approach.

Results: Fifty-five clinical staff participated in the study. Three themes emerged from the data (1) Risk assessment and planning: "The powder keg," (2) Innovative evolution of roles and responsibilities, and (3) Pandemic response and capacity. In the early phases of the pandemic, participants reported varying levels of risks for dying. However, most participants adapted to practice changes and became innovative in their roles over time. Hierarchies were less relevant during care delivery, whereas team collaboration became crucial in managing workforce capacity.

Discussion: As the pandemic progressed, staff preparedness evolved through a trial-and-error approach.

Conclusions: The pandemic is evolving as is clinical staff preparedness to meet the demands of a pandemic. In order to get a grasp on the crisis, clinical staff relied on each other and resorted to new workarounds.

(c) 2020 Association for Professionals in Infection Control and Epidemiology, Inc. Published by Elsevier Inc. All rights reserved.

\section{BACKGROUND}

Since the emergence of the SARS-CoV-2 (COVID-19) pandemic in December 2019, COVID-19 cases have increased rapidly on a global level with the largest numbers of cases being in the United States (US). ${ }^{1}$ Clinical staff have been on the frontlines of the pandemic response, delivering care amidst strained health care resources, limited workforce supply, and fluctuating clinical protocols for COVID19 treatment. In tandem with federal, state, and local efforts to contain and mitigate the virus, researchers have tried to understand best isolation practices, contact tracing procedures, and transmissions patterns. ${ }^{2-5}$ Yet little attention has been paid to clinical staff

\footnotetext{
* Address correspondence to Sainfer Aliyu, PhD, MSEd, MHPM, RN, MedStar Washington Hospital Center, 110 Irving St NW, Washington, DC 20010.

E-mail address: sainfer.e.aliyu@medstar.net (S. Aliyu).

Conflicts of interest: None to report.
}

preparedness and response. ${ }^{4,6,7}$ This oversight has limited the development of robust and actionable plans for addressing public health emergencies now and in the future. ${ }^{8}$ Research in this area is needed to support clinical staff preparedness for public health emergencies including the current COVID-19 pandemic. Therefore, the purpose of this study was to obtain various clinical staff perspectives about preparedness to meet the demands for care during the early phase of the COVID-19 pandemic.

\section{METHODS}

\section{Study design and setting}

In this qualitative descriptive study, we conducted interviews with clinical staff between March 2020 and April 2020. The setting was a large tertiary academic urban hospital center located in the northeast region of the United States. 


\section{Selection of participants}

A convenience sampling technique was used to recruit clinical staff caring for patients diagnosed with COVID-19, including physicians (MDs), registered nurses (RNs), patient care technicians (PCTs), respiratory therapists (RTs), and pharmacists. Using a snowball sampling technique, existing participants were asked to refer colleagues that would potentially be interested in participating. Seventy-five clinical staff was approached and 55 recruited based on availability, willingness to participate and data saturation. Clinical staff were eligible to participate if they were (1) 18 years of age or older, (2) English-speaking, (3) able to complete a one-time telephone or in-person interview, and (4) caring for a patient diagnosed with COVID-19.

\section{Data collection instruments}

Two instruments were developed to facilitate data collection. The first was a semistructured interview guide for use during the interviews. This guide was informed by the Resilience Framework for Public Health Emergency Preparedness. ${ }^{9}$ Questions were open-ended, evaluated by study team members for clarity and content, and pilot tested with 3 clinical staff. A demographic questionnaire was additionally developed to assess age, race, gender, education, years in profession, unit, and years in current position.

\section{Procedures}

One-on-one in-depth interviews were conducted by the principal investigator over a three-week period. All interviews were conducted in-person, in a private location at the hospital site and at a time convenient to the participant. Informed written consent was obtained prior to the start of the interviews. Interviews lasted approximately 15-25 minutes and were audio-recorded. Data collection continued until thematic saturation was achieved. Interviews were professionally transcribed, reviewed for accuracy, and identifiers removed. The Institutional Review Board of MedStar Health Research Institute approved the study.

\section{Data analysis}

\section{Resilience Framework for Public Health Emergency Preparedness}

The Resilience Framework for Public Health Emergency Preparedness informed the analysis of the data and interpretation of the findings. The framework includes 11 essential elements of public health emergency preparedness: (1) governance and leadership, (2) planning process, (3) collaborative networks, (4) community engagement, (5) risk analysis, (6) surveillance and monitoring, (7) practice and experience, (8) resources, (9) workforce capacity, (10) communication, and (11) learning and evaluation. These elements represent the upstream approach to readiness for a public health emergency; for this study, the elements were applied to exploring clinical staff's preparedness for the COVID-19 pandemic. $^{9}$

We followed the Consolidated Criteria for Reporting Qualitative Research 32-item checklist to ensure a standard method for reporting qualitative studies. ${ }^{10}$ Analysis of the transcripts began iteratively with data collection. First, 3 coders (AN, JT, and KS) collaboratively drafted a definition of each of the themes based on the Resilience Framework for Public Health Emergency Preparedness. After these definitions were drafted, the same three coders, working independently, read a random subset of 7 transcripts to obtain a shared understanding of the relevant codes and identify categories based on elements within the framework. These transcripts were subsequently coded using a directed content analysis approach. ${ }^{11}$ The research team met to discuss discrepancies, ensure consensus in interpretations, and identify where definitions needed to be refined. The coding team then repeated the same process with another random subset of 7 transcripts. Twenty-five percent of the data were double coded in total and discrepancies were resolved during routine meetings until a Kappa agreement of $90 \%$ or greater was achieved. After development of the final codebook, the remaining transcripts were independently coded by 5 investigators. Weekly team meetings were held to reconcile data. NVivo 12 qualitative data analysis software was used to organize the data and ensure dependability. The research team worked together to develop themes that reflected the data emerging within the constructs of our framework.

\section{RESULTS}

Data saturation was achieved after 55 interviews with RNs $(n=21)$, PCTs $(n=13)$, MDs $(n=12)$, RTs $(n=5)$, and pharmacists $(n=4)$. There were no drop-outs. The majority of participants was African American (49\%) and ranged from 23 to 65 years old. Participants worked across a range of departments including the intensive care unit, emergency department, medical-surgical unit, RN float pool, respiratory therapy, and pharmacy. Over one-third of the sample had greater than 10 years of clinical practice experience (Table 1 ).

Three themes emerged from the data with the following descriptions: (1) Risk assessment and planning: "A powder keg" - Participants monitored the pandemic with increasing attention as the incidence spreads globally. They expressed a desire for more

Table 1

Participant characteristics $(\mathrm{n}=55)$

\begin{tabular}{|c|c|}
\hline Characteristics & Total n (\%) \\
\hline \multicolumn{2}{|l|}{ Clinical staff } \\
\hline Registered nurse & $21(38 \%)$ \\
\hline Patient care technician & $13(24 \%)$ \\
\hline Physician & $12(22 \%)$ \\
\hline Respiratory therapist & $5(9 \%)$ \\
\hline Pharmacist & $4(7 \%)$ \\
\hline \multicolumn{2}{|l|}{ Age category } \\
\hline $21-30$ years & $11(20 \%)$ \\
\hline $31-40$ years & $24(44 \%)$ \\
\hline $41-50$ years & $10(18 \%)$ \\
\hline$\geq 50$ years & $10(18 \%)$ \\
\hline \multicolumn{2}{|l|}{ Race/ethnicity } \\
\hline African American & $27(49 \%)$ \\
\hline White & $13(24 \%)$ \\
\hline Asian & $9(16 \%)$ \\
\hline Hispanic & $6(11 \%)$ \\
\hline \multicolumn{2}{|l|}{ Gender } \\
\hline Female & $41(75 \%)$ \\
\hline Male & $14(25 \%)$ \\
\hline \multicolumn{2}{|l|}{ Education level } \\
\hline Certification & $11(20 \%)$ \\
\hline Bachelor's degree & $27(49 \%)$ \\
\hline Master's degree & $4(7 \%)$ \\
\hline Doctoral degree & $13(24 \%)$ \\
\hline \multicolumn{2}{|l|}{ Years in profession } \\
\hline $0-5$ years & $18(33 \%)$ \\
\hline$>5-10$ years & $18(33 \%)$ \\
\hline$>10-15$ years & $9(16 \%)$ \\
\hline$\geq 15$ years & $10(18 \%)$ \\
\hline \multicolumn{2}{|l|}{ Years in current role } \\
\hline $0-5$ years & $29(53 \%)$ \\
\hline$>5-10$ years & $17(31 \%)$ \\
\hline$>10-15$ years & $5(9 \%)$ \\
\hline$\geq 15$ years & $4(7 \%)$ \\
\hline \multicolumn{2}{|l|}{ Department } \\
\hline Intensive care unit & $15(27 \%)$ \\
\hline Emergency department & $13(24 \%)$ \\
\hline Medical-surgical unit & $10(18 \%)$ \\
\hline Registered nurse float pool & $8(15 \%)$ \\
\hline Respiratory therapy & $5(9 \%)$ \\
\hline Pharmacy & $4(7 \%)$ \\
\hline
\end{tabular}


guidance and support related to personal protective equipment (PPE) use; (2) Innovative evolution of roles and responsibilities - Several participants reported adapting their care to minimize their likelihood of contracting the virus. Other participants explored staff-driven approaches to adapting to care, such as developing strategies to protect patient confidentiality; and (3) Pandemic response and capacity - Many participants expressed a greater feeling of teamwork. Hierarchies seemed to compress with a leveling of the traditional roles played by different professions. As participants gained experience in caring for COVID-19 patients and as leadership disseminated information, participants' confidence in their capacity to provide care increase.

\section{Risk assessment and planning: "A powder keg"}

From the early stages of the COVID-19 pandemic, participants reported monitoring global news to assess risk and predict their future role caring for patients with COVID-19. An RN described her early sense of alarm as "a powder keg ready to kick off" (RN). Prior to the first locally identified COVID-19 patient, there was variability in reported levels of concern. Some clinical staff reported a lack of fear and as if COVID-19 would be "like the common cold" (MD). Yet, as the surge began to spread across the United States and affect the local region, clinical staff reported an increased sense of alarm. One of the MDs described the rising COVID-19 statistics as such: "All of a sudden it went from zero to 100 a day and it's just like everything's shutting down... kind of almost in a span of an overnight. It's crazy" (MD).

Clinical staff perceptions of the pandemic's urgency prompted realizations and questions about their organization's ability to respond. "We keep hearing about this surge that hasn't hit yet, but it is going to get worse, and we keep going back and forth as it pertains to the low-key gossip about what's going to happen next. When are we going to run out of masks? We don't have assurances, and so we are all just kind of left in the dark about it" (RN). The desire for effective protocols and communication between clinical teams and administration were identified as key to planning and preparation. Yet, several clinical staff reported frustration with frequently changing policies guiding evidence-based care delivery. "The lack of consistency [in policy] is because it's not really scientifically-based, it's frustrating. I think it's more based on the supplies you have, the staff you have, more than anything else. That is not right" (MD). Several clinical staff reported a need for more communication about patient testing, staff resources such as PPE, patient assignments, and family visitors' policies.

The rapidly evolving knowledge about resources needed to prevent transmission and immediate availability of equipment and protocols prompted further concern. However, participants across departments expressed varying levels of confidence in their preparedness to effectively deliver care to patients with COVID-19. An MD described his preparedness as, “About as ready as I can be. I think it's something that's new that everyone's dealing with together" (MD). Pharmacists also reported feeling adequately prepared for PPE donning and doffing: "We deal with hazardous drugs. We prepare IV solutions, so we're familiar with negative rooms, with clean rooms. We're familiar with garnering the process of the scrubs and the gloves, scrubs to cover your feet, the full gown, the face mask, the N95 mask with eyeglasses.... it's not totally entirely new. So, I think just because of our profession, we're kind of prepared for that" (Pharmacist).

Other participants expressed frustration with PPE access. "The PPE that we have access to is very conveniently located because of the fact that we have to save our supplies. So, we have to go to the manager and get our supplies, which is a bit of a hassle. It's all locked up except for gowns. So, gowns and masks we can get in front of the patient's rooms, but the N95, the caps, the boots, the surgical masks, they're all usually behind the desk or locked in a cabinet" (RN).
Innovative evolution of roles and responsibilities

Participants reported that over time, clinical care delivery processes and administrative leadership support evolved. There was a reported need for innovative changes when clinical teams lacked PPEs and were expected to care for a surge of patients without clear guidance for treatment protocols or a plan of care. A nurse recalled the SARS and Ebola outbreaks as pivotal moments in our nation's health care system noting differences to the current COVID-19 pandemic: "Once again, we are experiencing another outbreak, only this time it's a pandemic. We are not ready. We were more prepared for Ebola. I'm scared, not because I don't know how to do my job - I'm panicking because we are not ready. We have no PPEs, no one understands this disease and we did not receive the same training as we did when we prepared for Ebola. Did they forget! This is a joke" (RN).

The perceived lack of preparedness further prompted clinical staff to improvise through shared knowledge as a way to help solve unexpected challenges that the pandemic posed for the patients and the hospital. The novelty of the virus yielded a trial-and-error approach. "There is no standard of treatment. It's just too new. This disease is just too new. Everything is evolving. I feel the main reason why these things are changing is because they do not know exactly...No one knows what to do" (MD).

Several patient-care processes including discipline-specific roles changed. For example, the frequency of patient visits via telehealth increased in an effort to reduce clinical staff exposure to the virus. Several MDs and pharmacists reported increased remote e-consults, working from home, and communicating with colleagues via phone rather than in-person. In contrast, RTs denied large shifts in their role and reported, "It's pretty much the same. I haven't changed. I come from a climate where we had TB patients so it's not too much different with the exception of hearing about a 'cure'- so, yeah... nothing different" (RT).

On the contrary, RNs and PCTs reported increased patient exposure at the bedside compared to their clinical colleagues. Specifically, RNs reported additional nonclinical tasks that "required [RNs] to be in [the room] more" (RN). This was due in part to limitations in the responsibilities of other health care workforce to avoid viral exposure. A PCT explained, "The cleaning people, the environmental services. . .everybody wants to limit their exposure, so they're doing it less often" (PCT). In addition, an RN reported that, "dietary folks are not allowed in the rooms, so if I just gowned up, did all my care for my patient. I come out, and then, the meal - the trays arrive on the floor 15 minutes later. It's my job to re-gown up and deliver that tray to the patient" (RN).

Several RNs and nursing administrators also reported adapting nursing-specific care delivery processes to meet the immediate needs of the patients and fellow clinical staff. For example, a nursing supervisor shared an innovative method to maintain patient confidentiality by identifying patients under investigation or with COVID-19 as "blue patients." Anticipating patient needs, along with effective communication with prescribing providers, were reported as crucial aspects of care delivery reorganization performed by RNs and PCTs. "You [must] communicate with the doctors, just because. . to have a game plan before we go in, to anticipate what [the physicians will] order. You just bring in blood cultures, ice for lactate. You bring the COVID-19 swab. Bring in some Tylenol or have a runner outside to get you some Tylenol or whatever because $90 \%$, they're always febrile" (RN)

\section{Pandemic response and capacity}

Team collaboration and communication were reported as a central attribute of maintaining workforce capacity during the pandemic. Many participants described increased connection and a greater 
collaboration among coworkers. For example, a PCT reported: "[My coworkers] are very supportive. Everybody, today especially, I am very thankful to all the nurses. They are full of confidence. Everybody's helping each other...We are [friendlier] and more supportive now. . it's more attachment together now" (PCT). Another PCT said, "...we're all in this same scary adventure together" (PCT).

Traditional clinical hierarchies were also reported as less important during delivery of care. For example, PCTs corrected MDs for donning PPE inaccurately; and RNs and PCTs worked collectively as a 2-person team to "cluster care." There was a noted recognition of each worker's unique contribution as one RT described, "The cleaning crew, I constantly talk to them, for keeping the sanitation of the place, because without that, then everybody would be sick, so that's very important" (RT). An RN also shared, "This entire unit would fall apart if it were not for our techs. They are the blood, sweat, and tears of our team, and I love when our RNs are willing to listen and learn from them because it creates a sort of inter-dependence that we all really need right now, especially when we are isolated in the rooms" (RN). As processes and workflows evolved, teamwork evolved as well. There was an expanded role of infectious disease MDs working with all care teams. One MD described the process as the following: "There's an attending backup that is actively helping - not just waiting around for work - but actively helping with new COVID-19 econsults. There's a backup fellow that's doing the same, and we've had to recruit medicine residents to help with the workload. So, right now, we probably have three more people - three or four more people - working actively than we would in a non-pandemic situation" (MD).

Capacity building occurred concurrently with participants' role evolution. The amount and frequency of information coming from leadership increased, and many participants reported that such information increased their perceived ability to care for patients with COVID-19. For example, one RT reported an appreciation of the role of their director: "Our director is very good at updating us, what is the change, day-by-day and what we need to do. For example, if something comes up that is new or a therapy that we need to implement ...or what we can do to help the patient, and how to protect ourselves" (RT). A PCT also noted the helpfulness of regular emails, "My leaders have been very, very proactive about sending us information. We get anywhere from five to six emails a day" (PCT). Other participants shared an appreciation for the evolving practical knowledge about COVID-19 as they cared for more patients: "We're seeing more and more patients and getting a better understanding for ourselves, beyond the literature about the presentation and progression of the disease" (MD). A sense of confidence and preparedness emerged as time passed. A PCT stated, "This is my job and I have all the tools that I need to be able to do my job and I have been taught how to put on my PPE in a proper way and I know how to take my PPE off the right way" (PCT).

\section{DISCUSSION}

In this study, we explored clinical staff's perceptions of preparedness during the early stages of COVID-19 pandemic (March 2020April 2020). Participants reported uncertainty surrounding initial feelings of risk to themselves and their patients and partially attributed to the everchanging treatment and workforce safety protocols. Workforce roles evolved through innovative team-based care models to expand care capacity and align with available resources. Our study findings illuminate recommendations for preparing clinical staff for the current pandemic and future disaster-like events that require timely preparation and resource procurement.

Communication was key to maintain a sense of control in early stages of the pandemic. During preparations for the surge in cases, numerous clinical staff desired additional guidance including training specific to PPE. Individual- and system-driven information guiding clinical care delivery was warranted. Communication must instruct, inform, and motivate protective behavior, increase confidence in workforce, include timely updates, and dispel misinformation. Our findings align with previous evidence that disruptive actions (eg, increased handwashing and quarantines) in the care delivery setting yields heightened concerns and emotions. ${ }^{12}$ Pharmacists were more comfortable with the PPE use in comparison to all other groups of clinical staff, possibly related to the USP 800 Hazardous Drug-Handling in Healthcare Settings standards which they are required to follow. USP 800 provides guidance for fundamental practices and precautions for safe handling of hazardous drugs to minimize exposure to personnel. ${ }^{13}$ On the contrary, we observed differences in discipline-specific confidence levels and found that RNs and PCTs experienced more concern about PPE use. This may be due to the increased direct patient care that RNs and PCTs performed in comparison to other clinical staff, which instead increased remote patient care delivery to decrease COVID-19 exposure. Future research should investigate workforce initiatives that alleviate concern specific to knowledge and training preparation.

The approach to effective health care delivery during a pandemic is an evolving process. In our study, we noted clinical staff's trialand-error approach to innovate care processes driven by available PPE, concrete treatment protocols, and evolving disciplinary roles. The increased use of electronic communication using internal platforms was a pivotal support system for maintaining care delivery, ensuring timely communication, and tracking changing policies. Physicians and pharmacists increasingly performed remote e-consults, telehealth, and communication with colleagues by phone. There was less use, however, of Health IT applications reported by RNs and PCTs. Again, this may be due to the increased responsibilities of RNs and PCTs at the bedside. Researchers have previously noted that in previous disaster-like events, nursing roles appear to become more uncertain. ${ }^{14}$ The maintenance of effective and timely communication is key to defining clear role expectations.

Our findings also illuminated a compression of traditional workforce hierarchies as workers reported increased team-based coordination regardless of discipline. Some participants reported hierarchical changes to meet the demands for safety and capacity. For example, PCTs were comfortable correcting team members' incorrect use of PPE including RNs and MDs. Several states have recognized the need for workforce flexibility and are now flexible around licensure and scope of practice regulations, with governors issuing executive orders to temporarily modify scope of practice laws. ${ }^{15}$ Further, enhanced care environments were characterized by a greater perception of worth and value among team members. Although the clinical staff identified that ongoing change in institutional policies was initially frustrating, enhanced clarity of the viral etiology reduced worry. Clarity is vital as a poor understanding of the virus, including prevention and control measures, contributed to the infection of 3,000 clinical staff in Hubei, China, during the early stages of the pandemic. ${ }^{16}$ The provision of information to support capacity building including email updates with modifications to policies, procedures, and evidence-based practice, was viewed as helpful and supportive. When health care systems are not prepared to handle outbreaks like COVID-19, education and improved communication become critical. ${ }^{16}$ Clinical staff expressed appreciation for more concrete knowledge dissemination.

It is important to situate our results about clinical staff preparedness within the context of the hospital's emergency response. For example, hospital emergency response activities during the pandemic was led daily by the emergency preparedness team, which was expanded from 2 to 6 full-time administrative leaders with experience in public health emergency preparedness. The team was responsible for constructing hospital system-based responses and 
on-going daily training for emergency preparedness as defined by the US Department of Homeland Security, Federal Emergency Management (FEMA), the National Incident Management System (NIMS), and were aligned with FEMA's Homeland Security and Evaluation Program (HSEEP). In response to COVID-19, the incident command center was broadened from an administrator on-call response utilizing the NIMS structure to adding 2 executives and 3 administrators who were on-site 24/7. Their main function was to continuously assess hospital planning and preparedness by overseeing the following: communication efforts; bed-capacity; COVID-19 case volume; staffing needs; staff education and training; infection prevention compliance and surveillance; patient and staff safety; patient care delivery; and resources such as hospital protocols, equipment and supplies, and the employee assistance program. The hospital emergency response team and the clinical staff worked closely together to ensure optimal care delivery. However, our findings suggest a need for better integration between the hospital emergency response activities and clinical staff preparedness. Priority actions recommended during the COVID-19 response include the creation of a pandemic preparedness committee with representatives of all clinical disciplines, senior administrators, and support staff. ${ }^{17}$

Our findings highlight meaningful implications for sustainment of the clinical workforce especially during the early phases of the pandemic. First, findings suggest a need to broaden telehealth technology to support remote patient monitoring, data management, facilitate public health mitigation strategies, and preserve staff. For instance, telehealth delivery offers less exposure to COVID-19. Hence, these clinical staff were available to go into the hospital and provide care should their colleagues fall ill from COVID-19. Other investigators have reported the use of telehealth among quarantined providers, allowing them to continue providing care. ${ }^{15}$ Further, tele-ICU models can be expanded for specialists to e-consults at multiple sites. ${ }^{15}$ Second, in a rapidly changing landscape of practice protocols, public health guidance and discipline-specific responsibilities, it is key to secure capacity-building resources to support clinical staff early on. Clear, concise, and easily accessible knowledge must be disseminated to all workforce personnel regardless of discipline. Third, given the importance of workforce safety, there is a need for early education to enforce correct PPE use to alleviate personal risk concerns. This includes re-education of the donning and doffing of PPE to confirm staff are effectively protecting themselves. Data suggests substantial self-contamination risk occurs when doffing PPE, therefore re-education is critical. ${ }^{18}$ Lastly, innovative care delivery models such as clustering care can increase the workforce's ability to reduce unnecessary exposure and to promote team cohesiveness. Such guidance must strike a balance between training providers to limit unnecessary entry into patient rooms but also ensuring that patients and staff do not feel isolated or neglected. To ensure high-quality care, interprofessional and interorganizational collaboration must be a priority. A focus on team-based communication and collaboration, promoting an environment of mutual trust, respect, and a sense of belonging, and establishing standardized procedures are essential during a pandemic response. ${ }^{16}$ More research is needed to understand which approaches to patient care delivery, including team skill mix, are needed to optimize patient and system-wide capacity.

For health care workers in the United States and around the world, we identified related themes. ${ }^{16,19-21}$ Consistent with our findings, other qualitative investigations of the COVID-19 workforce identified the challenges of caring for patients, including the need for proper training with the everchanging understanding of viral etiology. ${ }^{19-21}$ Adapting and expanding their scope of practice was another similar theme. ${ }^{19}$ Major themes of other studies that were not identified in our study included a strong sense of duty to ensure their patients' optimal well-being and resilience as the key to overcoming challenges. ${ }^{16,19,20}$ Additionally, social stigma was an interesting finding for RNs in Iran. They experienced obvious and latent stigma in their communities, including the distancing of family members. ${ }^{21}$

There are limitations to our study. First, this study was limited to one hospital in the Northeast region of the United States. While it is similar to other academic medical centers in size and characteristics, organizational-specific policies and culture may be different. ${ }^{22}$ Also, our convenience sample of frontline clinical staff may hold varying perspectives than their clinical colleagues. However, we made efforts to recruit a representative interdisciplinary sample and employed theoretically-driven data analysis to allow for emergent themes.

\section{CONCLUSIONS}

COVID-19 had emerged as a significant global public health threat, with clinical staff being on the frontlines of pandemic response. Overall, our findings demonstrate that clinical staff's ability to respond to a pandemic is complex and is influenced by hospitals' leadership ability to effectively plan, communicate and drive system-wide change for health care delivery. In an emergent time of uncertainty, the compression of formal hierarchies enables clinical staff to deliver care in an "all hands on deck" approach to meet the demands for care. Evidence-based, robust approaches to public health emergency preparedness, with a focus on supporting the frontline clinical staff, can promote effective responses to both the current COVID-19 pandemic and future public health emergencies.

\section{References}

1. Centers for Disease Control and Prevention. Coronavirus Disease 2019 (COVID-19) Cases in U.S.. Atlanta, GA: Centers for Disease Control and Prevention; 2020. Available at: https://www.cdc.gov/coronavirus/2019-ncov/cases-updates/cases-in-us. html. Accessed October 6, 2020.

2. Pan A, Liu L, Wang C, et al. Association of public health interventions with the epidemiology of the COVID-19 outbreak in Wuhan, China. JAMA. 2020;323:1915-1923.

3. Paules C, Marston H, Fauci A. Coronavirus infections-more than just the common cold. JAMA. 2020;323:707-708.

4. Wang C, Ng C, Brook RH. Response to COVID-19 in Taiwan: big data analytics, new technology, and proactive testing. JAMA. 2020;323:1341-1342.

5. Wu Z, McGoogan J. Characteristics of and important lessons from the coronavirus disease 2019 (COVID-19) outbreak in China: summary of a report of 72,314 cases from the Chinese Center for Disease Control and Prevention. JAMA. 2020;323: 1239-1242.

6. Diao M, Zhang S, Chen D, Hu W. The novel coronavirus (COVID-19) infection in Hangzhou: an experience to share. Infect Control Hosp Epidemiol. 2020:1-2.

7. Brooks S, Webster R, Smith L, et al. The psychological impact of quarantine and how to reduce it: rapid review of the evidence. Lancet. 2020;395:912-920.

8. Wu J, McCann A, Collins K, et al. Coronavirus map: tracking the global outbreak. 2020. JAMA. 2020.. NYtimes.com. Available at: https://www.nytimes.com/interactive/2020/us/coronavirus-us-cases.html. Accessed May 14, 2020.

9. Khan Y, O'Sullivan T, Brown A, et al. Public health emergency preparedness: a framework to promote resilience. BMC Public Health. 2018;18:1-16.

10. Tong A, Sainsbury P, Craig J. Consolidated criteria for reporting qualitative research (COREQ): a 32-item checklist for interviews and focus groups. Int J Qual Health Care. 2007; 19:349-357.

11. Hsieh H, Shannon S. Three approaches to qualitative content analysis. Qual Health Res. 2005;15:1277-1288

12. Han P, Zikmund-Fisher B, Duarte C, et al. Communication of scientific uncertainty about a novel pandemic health threat: ambiguity aversion and its mechanisms. $J$ Health Commun. 2018;23:435-444.

13. USP General chapter $<800>$ hazardous drugs-handling in healthcare settings. USP 40-NF 35 Second Supplement, 2017. Available at: https://www.usp.org/sites/ default/files/usp/document/our-work/healthcare-quality-safety/general-chapter800.pdf. Accessed August 12, 2020.

14. Stirling B, Hatcher J, Harmston J. Communicating the changing role of a nurse in an epidemic: the example of the MERS-CoV outbreak in Saudi Arabia. J Healthc Commun. 2017;2:1-6.

15. Chen C, Pittman P, Westergaard S, et al. Emerging health workforce strategies to address COVID-19. Health Aff Blog. 2020

16. Lin $\mathrm{Q}$, Luo D, Haase JE, et al. The experiences of health-care providers during the COVID-19 crisis in China: a qualitative study. Lancet Glob Health. 2020;8:e790e798.

17. Toner E and Waldhorn R. What US hospitals should do now to prepare for a COVID-19 pandemic. Clinicians' Biosecurity News $>$ JHSPH Center for Health Security February 27, 2020.

18. Phua J, Weng L, Ling L, et al. Intensive care management of coronavirus disease 2019 (COVID-19): challenges and recommendations. Lancet Respir Med. 2020;8:506-517. 
19. Sun N, Wei L, Shi S, et al. A qualitative study on the psychological experience of caregivers of COVID-19 patients. Am J Infect Control. 2020;48:592-598.

20. Galehdar N, Toulabi T, Kamran A, Heydari H. Exploring nurses' perception of taking care of patients with coronavirus disease (COVID-19): a qualitative study. Nurs Open. 2020;00:1-9.
21. Sadati A, Zarei L, Shahabi S, et al. Nursing experiences of COVID-19 outbreak in Iran: a qualitative study. Nurs Open. 2020;00:1-8.

22. Jun J, Kovner C, Dickson V, Stimpfel A, Rosenfeld P. Does unit culture matter? The association between unit culture and the use of evidence-based practice among hospital nurses. Appl Nurs Res. 2020;53:442-451. 